\title{
BMJ Open Patient participation in free cataract surgery: a cross-sectional study of the low-income elderly in urban China
}

\author{
Haotian Lin, Duoru Lin, Erping Long, Haofeng Jiang, Bo Qu, Jinzhu Tang, \\ Yingfen Lin, Jingjing Chen, Xiaohang Wu, Zhuoling Lin, Xiaoyan Li, Zhenzhen Liu, \\ Bo Zhang, Hui Chen, Xuhua Tan, Lixia Luo, Yizhi Liu, Weirong Chen
}

To cite: Lin H, Lin D, Long $\mathrm{E}$, et al. Patient participation in free cataract surgery: a crosssectional study of the lowincome elderly in urban China. BMJ Open 2016;6: e011061. doi:10.1136/ bmjopen-2016-011061

- Prepublication history and additional material is available. To view please visit the journal (http://dx.doi.org/ 10.1136/bmjopen-2016011061).

$\mathrm{HL}, \mathrm{DL}, \mathrm{EL}$ and $\mathrm{HJ}$ contributed equally.

Received 6 January 2016 Revised 24 February 2016 Accepted 21 March 2016

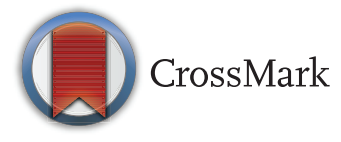

The State Key Laboratory of Ophthalmology, Zhongshan Ophthalmic Center, Sun Yat-sen University, Guangzhou, Guangdong, People's Republic of China

Correspondence to Professor Weirong Chen; chenwr_q@aliyun.com

\section{ABSTRACT}

Objectives: To explore the characteristics of the lowincome elderly who underwent free cataract surgery and to determine the degree of patient satisfaction with the free cataract surgery programme in urban China.

Methods: A free cataract surgery management workflow was designed as a poverty relief project in Guangzhou. In this study, participants who underwent free cataract surgery between January and August 2014 received a telephone interview based on a structured questionnaire. Data were collected on patient demographics, resources, health conditions, reasons for undergoing the free surgery and overall evaluation of the free cataract surgery programme.

Results: Among the 833 participants, the mean surgical age was $76.85 \pm 7.46$ years $(95 \% \mathrm{Cl} 76.34$ to 77.36 ), and the male to female ratio was $385: 448$. The majority $(94.31 \%, 746 / 791)$ of patients resided in the main urban districts. Patients underwent surgery $61.08 \pm 60.15$ months ( $95 \% \mathrm{Cl} 56.17$ to 66.00$)$ after becoming aware of the cataract, although $66.83 \%$ of them reported that their daily lives were influenced by cataracts. Only $21.5 \%$ of the respondents underwent physical examinations that included regular eye screening, and only $6.30 \%$ were highly educated patients. Financial problems were the primary reason cited by patients for participating in the free surgery programme. Those patients with a monthly family income of 1000-2999¥ (US\$161-482) per capita constituted the largest patient population. The free clinics in the parks and the free cataract surgery were highly rated (9.46 and 9.11 of 10 points) by the beneficiaries.

Conclusions: The telephone survey revealed a high level of patient satisfaction regarding the free cataract surgery programme. Most of the patients who participated in the programme resided in major urban districts and had poor health awareness and a low level of education. The information provided by this study is crucial for improving and expanding the management of free cataract surgery programmes. Trial registration number: NCT02633865; Postresults.

\section{Strengths and limitations of this study}

- This is the first telephone survey to summarise the detailed patient characteristics of particularly the low-income community in urban China; most of the participating patients resided in the major urban districts and possess poor health awareness and a low level of education.

- The information provided by this survey is crucial for improving and expanding the management of free cataract surgery programmes.

- There may be some under-reporting as some participants and those who refused to respond may have been reluctant to report their low income and education levels although we had notified all respondents that their responses would be protected with strict confidentiality prior to the formal interview.

- This study describes the characteristics of only the low-income cohort that underwent the free cataract surgery in the programme; the characteristics of the low-income patients who did not undergo or have not yet undergone the free cataract surgery represent an area for future research.

\section{INTRODUCTION}

Age-related cataracts remain the leading cause of treatable blindness in China. ${ }^{12}$ The prevalence of cataract blindness continues to increase due to the growing elderly subpopulation. ${ }^{3}$ Surgery is the only available treatment; however, the cataract surgical rate (CSR) in China remains low (1125 cases per million per year) ${ }^{4}$ relative to that of affluent countries $(6000 \text { cases per million per year })^{5}$ and some developing areas (2672 cases per million per year). ${ }^{6}$ Studies have reported that the primary barrier to cataract surgery for individuals is financial difficulty and that this challenge could be efficiently addressed by reducing the surgical fee $^{1}$ or providing free cataract surgery. ${ }^{7}{ }^{8}$ However, the prices 
of medicines and medical services in China, and the availability and affordability of medicines and medical services for China's poor, especially the urban poor, require further improvement ${ }^{9} 10$ according to the standards developed in May 2003 by the WHO in collaboration with Health Action International (HAI). ${ }^{11}$ Although free cataract screening ${ }^{12-14}$ and surgery programmes ${ }^{7}$ have been widely implemented in rural areas of China in conjunction with a new national healthcare reform that was officially launched in 2009 to tackle high medical expenses, including the promotion of free medical treatments, ${ }^{9}{ }^{15}$ free cataract surgery programmes have rarely been implemented in financiallychallenged urban China. Even in Guangzhou, one of the largest metropolises in China, many low-income cataract patients, a neglected cohort, continue to need cataract surgery. A programme entitled 'Care for your eyes, lighten your life', jointly sponsored by the People's Government of Guangzhou Municipality, the Bureau of Civil Affairs of Guangzhou Municipality, and the Zhongshan Ophthalmic Center (ZOC), was initiated in Guangzhou in 2012 to implement a programme of providing free clinics in parks and free cataract surgery targeting China's impoverished urban population. ${ }^{16}$ After 3 years of operation, the management of this programme has been optimised, and approximately 4000 cataract surgeries have been performed on the lowincome elderly.

Although there are a large number of poor communities throughout the country, free cataract surgery programmes targeting poor urban China are restricted to several metropolises due to limited medical resources and social overlook. Understanding the level of patient satisfaction regarding the free cataract surgery programmes and the patient characteristics of this neglected community may contribute to the improvement and further expansion of the management of free cataract surgery programmes. By conducting this telephone survey, we aimed to identify the characteristics of the low-income patients who underwent free cataract surgery, including patient demographics, resources, health conditions, reasons for participating in the free surgery, and overall evaluation of the free cataract surgery programme.

\section{METHODS}

\section{Patients}

A free cataract surgery programme was jointly initiated by the People's Government of Guangzhou Municipality, the Bureau of Civil Affairs of Guangzhou Municipality, and the ZOC in May 2012. Guangzhou citizens who were candidates for this activity were confirmed to be (1) aged $\geq 50$ years with low income and who rely on social subsidies, or (2) laid-off workers, or (3) handicapped people, or (4) old Red Army or families of revolutionary martyrs and servicemen. All participants with presenting visual acuity (PVA), unaided visual acuity or aided visual acuity with walk-in optical correction $^{7}$ of $\leq 20 / 50$ in either eye without any severe eye diseases except cataract were included. The eligible participants successively experienced the following steps: preliminary screening, preoperative evaluation, preoperative examination, preoperative preparation, operation, and postoperative follow-up visit. ${ }^{16}$ A total of approximately 4000 low-income patients benefited from this free cataract surgery programme, including 1862 beneficiaries in the focus group of the present study, from September 2013 to August 2014. Of the 1862 patients, approximately $10 \%(185 / 1862)$ had preoperative PVA worse than finger count, and the mean PVA of the remaining $90 \%$ $(1677 / 1862)$ was approximately $20 / 100$. As outlined in figure 1, we conducted a telephone survey of 1019 patients who could be contacted by telephone and had undergone free cataract surgery between January and August 2014. Those patients who were not successfully contacted ( $>3$ attempts at different time points), who refused to participate, who failed to understand the conditions of the interview or suffered deafness were excluded. All patients in the cohort had been recruited from the free clinics in four parks located in Guangzhou and had undergone phacoemulsification with intraocular lens implantation at least 6 months ago. This study was registered in ClinicalTrials.gov registration (NCT02633865), and was approved by the Human Research Ethics Committee of the ZOC, Sun Yat-sen University. All procedures were consistent with the tenets of the Declaration of Helsinki and were performed after obtaining oral informed consent (provision of written consent were exempted by the IRB-ZOC-SYSU) from all respondents in the telephone prior to the formal interview.

\section{Interview methods and questionnaire}

A trained interviewer $(\mathrm{HJ})$ conducted the telephone interviews based on a structured questionnaire. All interviews involved hands-free communication, and all the responses by each respondent were repeated by the interviewer and confirmed by two other independent examiners (ZL and XL) to reduce the risk of judgment bias. Prior to each interview, all of the respondents were clearly informed that their responses would be protected with strict confidentiality. The structured questionnaire (acquired in online supplementary files 1 and 2) for the free cataract surgery programme was designed and revised by the Team of Chinese Cataract Poverty Alleviation Special Programme. The questionnaire comprised of 17 questions that were further divided into five subsections: patient demographics (number 1-3), patient resources (number 4-6), health conditions (number 7-12), reasons for choosing the free surgery (number 13-15), and overall evaluation of the free cataract surgery programme (number 16-17). The reliability and validity of the questionnaire was approved by statistical and ophthalmologic experts, and was used after pilot testing of telephone interview procedures on 50 


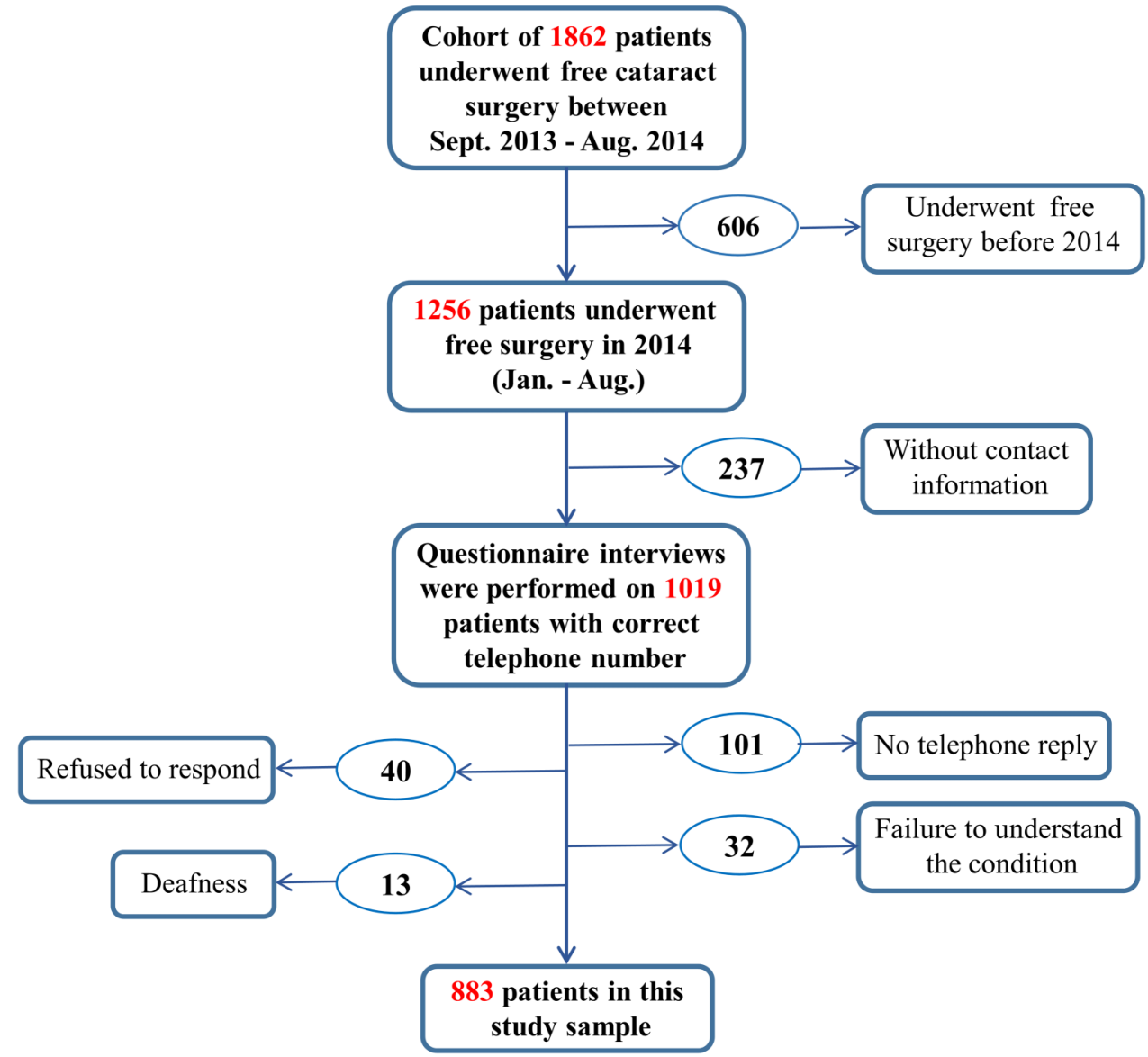

Figure 1 Flow diagram of the components of the telephone interview regarding the free cataract surgery.

participants (21 men and 29 women). In the pilot test, all telephone interviews were completed within 5-8 min with good feasibility.

\section{Statistical analysis}

All data were entered into Microsoft Excel 2010 spreadsheets (Microsoft Corp., Redmond, Washington, USA) and then sorted and analysed by two researchers (HL and DL), and finally mutually checked. Statistical analysis was performed using the Statistical Package for the Social Sciences (SPSS V.19.0, Chicago, Illinois, USA). Absolute frequency (n) and relative frequency (\%) were calculated for the qualitative variables, and the mean and SD (mean \pm SD) were calculated for the quantitative variables. The difference in PVA between the preoperative patients from September 2013 to August 2014 and the postoperative patients from January to August 2014 were analysed using a $\mathrm{t}$ test for independent samples. The relationships between the frequency of preoperative physical examination and household income, education level, presence of cataracts and presence of comorbid diseases were evaluated by multinomial logistic regression analysis. Multiple linear regression was used to analyse the relationship between delayed surgical treatment and education level or family income. All statistical tests were two-tailed, and a $\mathrm{p}$ value $<0.05$ was considered statistically significant.

\section{RESULTS}

In the telephone survey, 833 successful interviews were conducted among the 1019 patients (81.75\%) who underwent free cataract surgery in 2014 (from January to August) and could be contacted by telephone. The remaining patients were not interviewed due to no telephone reply $(101,9.91 \%)$, refusal to respond (40, $3.93 \%$ ), failure to understand the conditions of the interview $(32,3.14 \%)$, or deafness $(13,1.28 \%)$. Most of the successful respondents lived with children (52.14\%, 427/ 819 ) and (or) their spouse $(50.06 \%, 410 / 819)$. The mean age at surgery was $76.85 \pm 7.46$ years $(95 \%$ CI 76.34 to 77.36) (range, 50-102 years), and the male to female ratio was 385:448. Free cataract surgeries had been performed approximately 9 months before the survey $(9.14$ \pm 6.41 months in the right eye, $9.07 \pm 6.80$ months in the left eye). The mean postoperative PVA value was 20/33, with $93.08 \%(673 / 723)$ of patients having a PVA $\geq 20 / 60$; this result represented a significant improvement compared to the preoperative level $(<20 / 100)(\mathrm{p}<0.001)$.

Most of the patients $(94.31 \%, 746 / 791)$ resided in major urban areas, including Yuexiu, Haizhu, Liwan, 


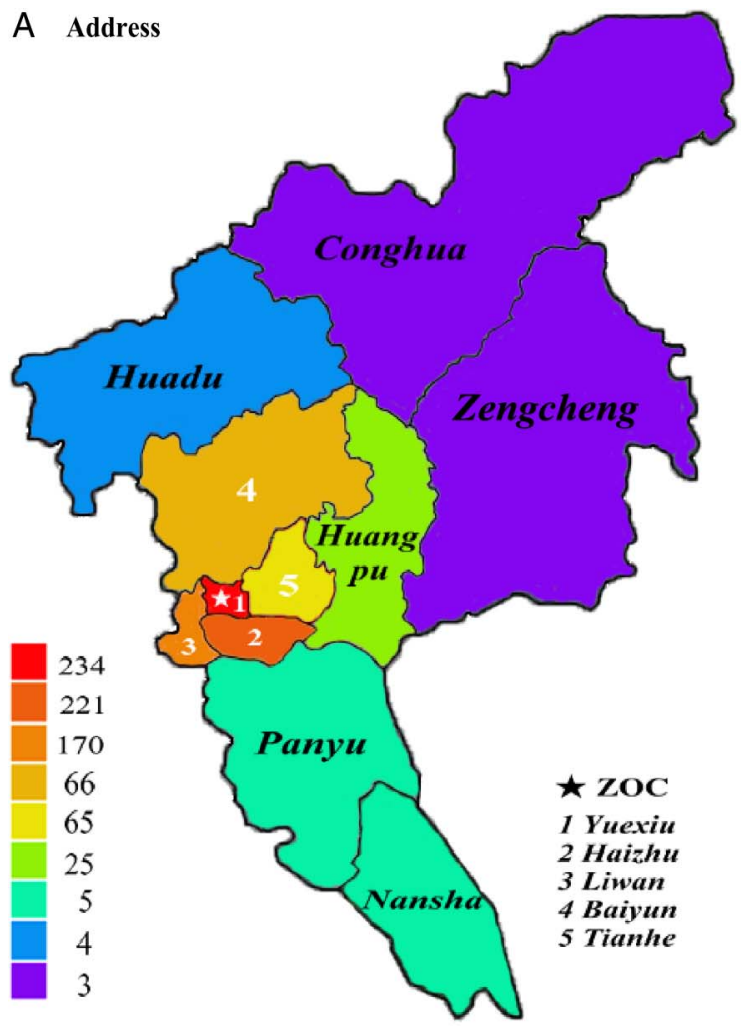

B Parks in which patients participated in the free clinics

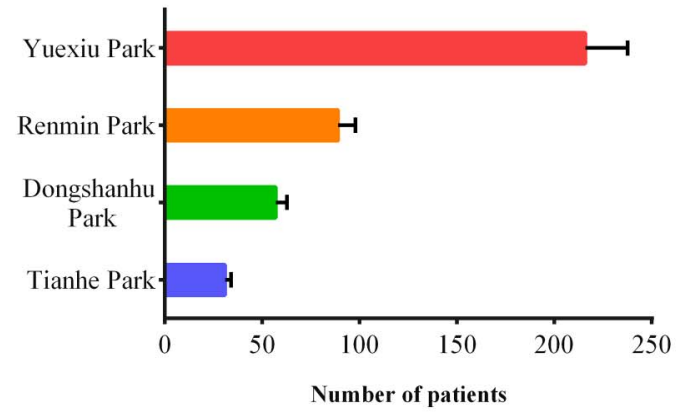

C Avenues to know

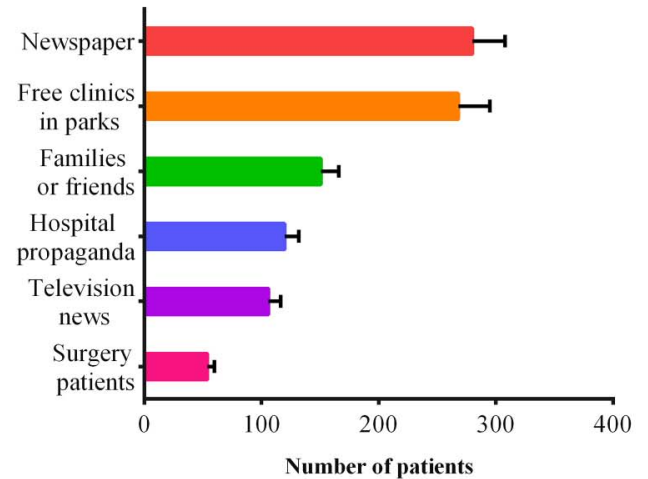

Figure 2 Patient resources. (A) Most of the patients (94.31\%) were residents of the major urban areas, including Yuexiu District, Haizhu District, Liwan District, Baiyun District and Tianhe District. (B) More than 90\% of the patients were recruited from the three parks (Yuexiu Park, Renmin Park and Dongshanhu Park) located in Yuexiu District. (C) The newspaper and the free clinics in the parks were the two most important sources of information concerning the free cataract surgery. ZOC: Zhongshan Ophthalmic Centre; Error bar: $10 \%$ of the value.

Baiyun and Tianhe districts (figure 2A). Among the patients from whom data were available, $92.11 \%$ (362/ 393) who had undergone the free cataract surgery had been recruited from the following three parks located in Yuexiu District: Yuexiu Park (54.96\%, 216/393), Renmin Park $(22.65 \%, 89 / 393)$, and Dongshanhu Park (14.5\%, $57 / 393$ ) (figure 2B). The free clinics in the parks were the second most important source of information concerning the free cataract surgery; the newspaper was the most important source (figure 2C).

Patients had undergone cataract surgery at an average of $61.08 \pm 60.15$ months (95\%CI 56.17 to 66.00 ) after becoming aware of the cataract, although $66.83 \%$ (548/ 820) of these patients reported that their daily lives had been moderately or severely influenced by poor vision (figure 3A). Furthermore, $30.31 \%(251 / 828)$ of the respondents did not know when the cataract first appeared. The health conditions of the patients are presented in figure 3. Of the patients, $46.47 \%$ were suffering from high blood pressure, $15.26 \%$ were diabetic and $12.94 \%(106 / 829)$ had been diagnosed with other diseases such as coronary heart disease and stomach trouble. However, figure 3B shows that only $21.5 \%$ (178/ 826) of the patients had undertaken regular physical examinations that included eye screening, and $25.06 \%$ $(207 / 826)$ of the patients had not had any physical examinations in the past 5 years before the free cataract surgery due to feeling slightly ill without significant effects on their daily life, not feeling ill, high medical expenses or other reasons as listed in figure 3C. In addition, as shown in figure 3D, the education level of the respondents was generally low: nearly $80 \%$ (661/826) had received only primary education (below the 9-year compulsory education), and only $6.30 \%$ (52/826) were highly educated (college/university education or above). The results of the multinomial logistic regression analysis revealed that those patients who did not feel ill or felt only slightly ill were more likely to refuse any physical examination $\left(\mathrm{P}<0.001, \chi^{2}=23.51, \mathrm{OR}=2.41,95 \% \mathrm{CI}\right.$ 1.69 to 3.45$)$. Patients with moderate to high level of education (high school or above) were more inclined to undergo periodic physical examinations $(\mathrm{p}<0.001$, $\chi^{2}=17.63, \mathrm{OR}=0.40,95 \%$ CI 0.26 to 0.61 ).

Figure $4 \mathrm{~A}$ reveals that financial problems were the most common reason offered by patients for postponing surgery until the free surgery was available; lack of awareness and fear of surgery were the next most commonly cited factors. Similarly, financial problems were the most common factor motivating patients to participate in the free surgery; programme advertisement and the reputation of the ZOC were additional important influencing factors (figure 4B). Those patients who had 
A Influenced by poor visual acuity

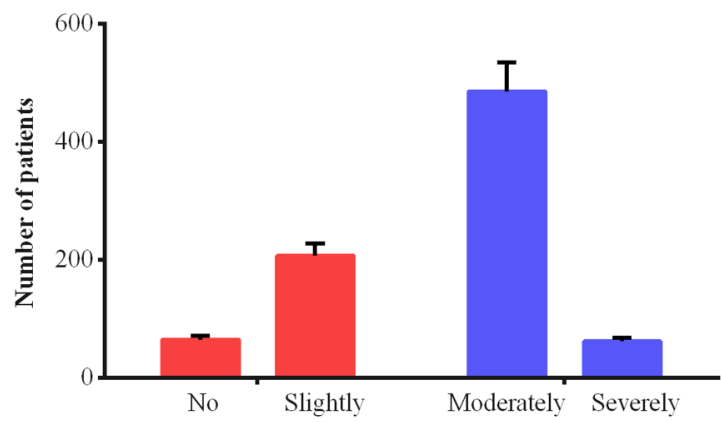

C Reasons for failure to the physical examinations

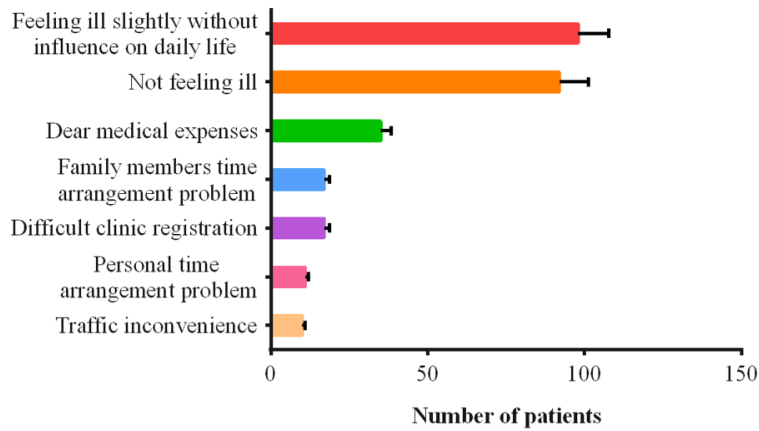

B Physical examinations in the last five years

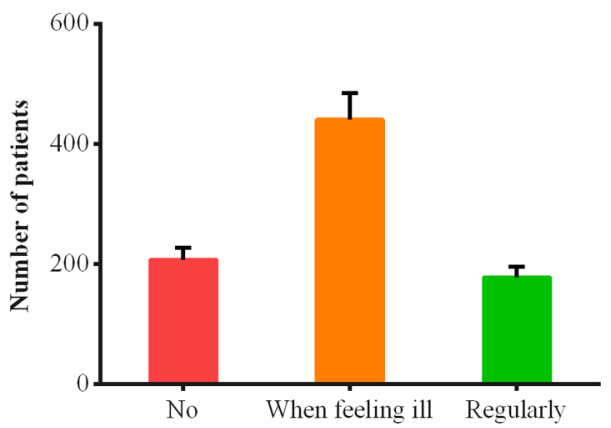

D Education level

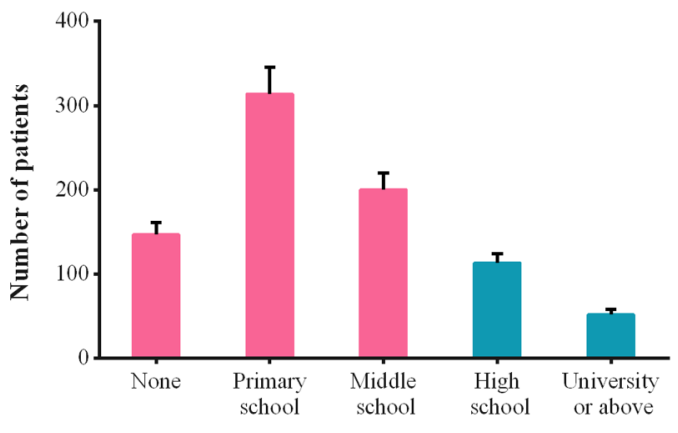

Figure 3 Health conditions and education level of the respondents. (A) Approximately $2 / 3(66.83 \%)$ of patients reported that they were influenced by poor visual acuity before the free cataract surgery. (B) only $21.5 \%$ of the patients underwent regular physical examinations including eye screening. (C) lists the reasons provided by those who failed to undergo the physical examinations; feeling slightly ill without significant effects on daily life and not feeling ill were the two most significant factors. (D) reveals that the education level of the respondents was generally low; nearly $80 \%$ had received only primary education. Error bar: $10 \%$ of the value.

a monthly family income of 1000-2999¥ (US\$158-472, $6.35 ¥=$ US $\$ 1$ ) per capita constituted the largest patient proportion $(52.13 \%, 428 / 821)$; only $5.72 \%(47 / 821)$ of the patients had a monthly family income greater than $5000 ¥$ (US\$789) per capita (figure 4G). The multinomial linear regression analysis revealed that patients with low education levels (below the 9-year compulsory education $)(\mathrm{p}=0.032, \mathrm{t}=-2.14, \mathrm{~B}=-4.88)$ or low family income $(<3000 ¥$, US $\$ 472) \quad(\mathrm{p}=0.059, \mathrm{t}=-1.89, \mathrm{~B}=-6.69)$ were more likely to (or tended to) delay surgical treatment (figure 4D).

Patients were requested to grade their level of satisfaction with the free clinics in the parks and the free surgery on a scale of $0-10$; the average scores were $9.46 \pm 1.06$ and $9.11 \pm 1.23$, respectively.

\section{DISCUSSION}

\section{Principal findings}

The results of this study clarified the characteristics of patients who benefited from the free cataract surgery programme. All patients underwent uneventful surgery with an increased postoperative PVA $(93.08 \%$ of patients had $\mathrm{PVA} \geq 20 / 60$ ), which exceeds the WHO guidelines for cataract surgery outcomes (at least $80 \%$ of eyes should have a 12 -week postoperative PVA $\geq 20 / 60) .{ }^{17}$
The free clinics in the parks and the free cataract surgery were highly rated (9.46 and 9.11 out of 10 points) by the beneficiaries. Most of the patients reside in the major urban districts of Guangzhou close to the ZOC, and newspapers and the free clinics in the parks were the two main avenues through which patients were informed of the free cataract surgery. Approximately $80 \%$ of the impoverished cohort had received only primary education. Furthermore, the health awareness of these patients was poor. Only $1 / 5$ underwent regular physical examinations before this free surgery programme, including eye screening, despite being in poor health and suffering from high blood pressure, diabetes mellitus or coronary heart disease. Finally, financial problems were the leading reason provided for why patients did not pursue surgery until free surgery was available and for patient participation in the free surgery programme.

\section{Interpretation and comparison with other research}

The coverage of this free cataract surgery programme needs to be expanded. Our results indicate that the free clinics in the park are one of the most important avenues through which people are informed of the free cataract surgery programme, possibly due to the healthy, urban lifestyle of the retired elderly who enjoy 
A Reasons for not doing surgery until this activity

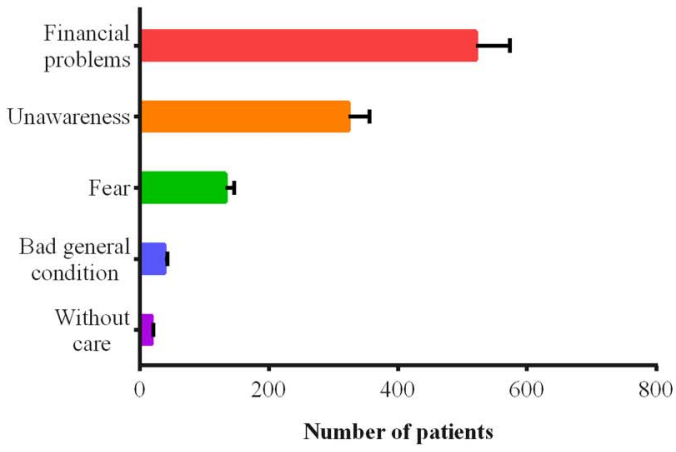

C

Family income

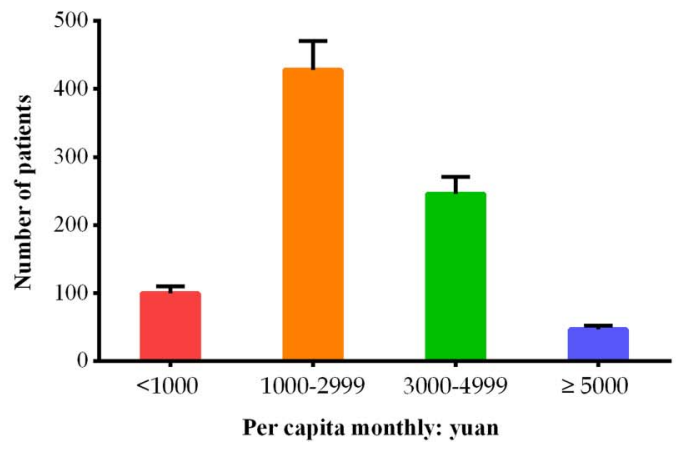

B
Reasons for participating in the free surgery activity

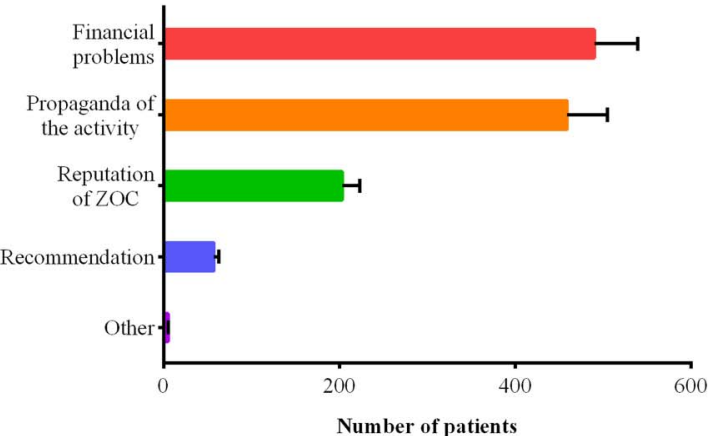

D

Relationship between delayed treatment
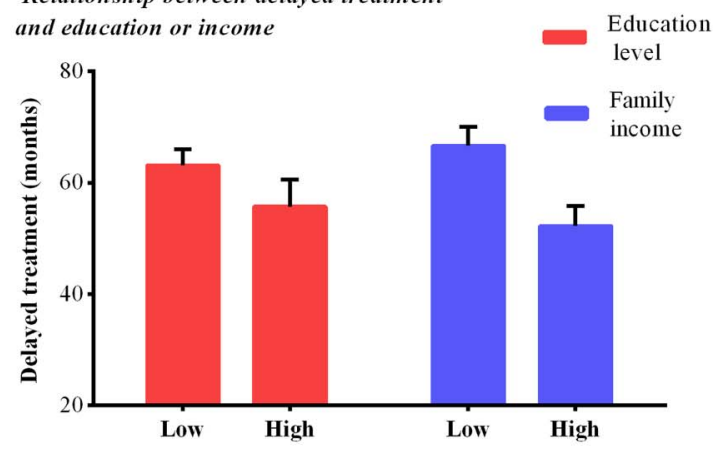

Figure 4 Reasons for patient participation in the surgery programme and patient household income distribution. (A and B) Financial problems were the leading reason provided for both why patients did not undergo surgery prior to the availability of the free surgery and why patients participated in the free surgery programme. (C) Those patients with a monthly family income of 1000-2999¥ (US\$158-472, 6.35¥=US\$1) per capita constituted the largest patient population (52.13\%). (D) Patients with a low education level (below the 9-year compulsory education) and low family income $(<3000 ¥$, US\$472) were more likely than other patients to delay surgical treatment. Error bar: $10 \%$ of the value in (A-C); SE in (D).

participating in sports in the parks each morning. ${ }^{18}$ Although the free cataract surgery programme is directed primarily at the low-income elderly throughout Guangzhou via the multichannel information disseminated throughout the entire city, including newspapers, television and WeChat (one of the largest standalone messaging apps in China), ${ }^{19}$ nearly $80 \%$ of the participating patients resided in the major urban districts in Guangzhou close to the ZOC. Newspapers represent the most common medium by which patients were informed of the free cataract surgery, indicating that the eligible candidates can be informed even if residing in remote districts of Guangzhou. We do not know the precise reasons for the low patient proportion from the remote districts, but the long distance between their district and the ZOC may be a major obstacle according to previous reports. ${ }^{20}$ For example, a patient living in Zengcheng district, which is an outskirt area more than $71 \mathrm{~km}$ (as calculated by Google maps) from the ZOC in Yuexiu District, would be required to must pay nearly $500 ¥$ (US $\$ 80$ ) to travel by taxi or to invest more than $8 \mathrm{~h}$ of travel time if travelling by public transport for a round trip.

The awareness of health in this low-income cohort is poor and should be increased. Only $1 / 5$ patients participated in regular physical examinations, including eye screening, and $1 / 4$ respondents did not pursue any physical examinations due to feeling only slightly ill without any significant effects on daily life or due to not feeling ill. In the multinomial logistic regression analysis, we also found that the patients who did not feel ill or who felt only slightly ill were more likely to refuse any physical examinations. However, $63.25 \%$ of the respondents were diagnosed with systematic diseases, and the prevalence of high blood pressure (46.47\%) and diabetes $(15.26 \%)$ in this cohort was much higher than that in the Chinese population $\left(25.2 \%\right.$ and $9.7 \%$, respectively). ${ }^{21}$ Furthermore, 2/3 of patients believed that their daily lives were moderately or severely influenced by poor vision. Schulze Schwering $e t a l^{22}$ previously reported that awareness of health services is significantly affected by the level of education and standard of knowledge of patients or relatives. We found that the education level of the respondents was generally low, with only $6.30 \%$ being highly educated (college/university or above) in contrast to the $9.9 \%$ of highly educated people nationwide. The results of the multinomial logistic regression analysis also revealed that highly educated patients were more inclined to undergo periodic physical examinations. 
Similar to previous reports, ${ }^{23}$ financial problems were the major reason provided for why patients participated in the free surgery programme. We found that the mean age at surgery of this low-income cohort was 76.85 years, which is much higher than the mean age of 70.56 years among patients who underwent paid cataract surgeries in the ZOC. ${ }^{24}$ Furthermore, patients had postponed the surgery for an average of 61 months after becoming aware of the cataract despite the report by $66.83 \%$ of them that their daily lives were moderately or severely influenced by poor vision. The results of the multiple linear regression analysis suggested that delayed surgical treatment may be related to the generally low education and family income level. Financial problems were the leading reason provided by patients for not having undergone the surgery before the free surgery programme was made available and the leading reason why patients participated in the free surgery activity. We found that those patients with a monthly family income of 1000-2999¥ (US\$158-472) per capita accounted for more than half of the patient population. The income of these families is only one-tenth that of the mean per capita income of Guangzhou households, according to the most recent data published on the website of Guangzhou statistical information. ${ }^{25}$ Thus, in addition to the surgical fee, addressing the indirect costs of patients and their companions, including transportation, accommodation, and loss of working time, may reduce the financial burden of patients and increase the CSR. ${ }^{22}{ }^{26}$ In addition, our results demonstrate that a lack of awareness of cataracts and a lack of awareness of the free surgery programme were the second most commonly provided reasons for why patients did not undergo surgery until the free surgery programme was made available and for why patients participated in the free surgery activity, a finding that is similar to the other reports. However, Burga et $a l^{20}$ noted that the lack of awareness of a disease is not a reason for not seeking surgery, and further concluded that a surgical package with all costs covered should be offered to the patients participating in the free cataract surgery programmes to increase the coverage of the activity.

\section{Implications for clinicians and policymakers}

To further expand the coverage of this free cataract surgery programme and to increase access to free cataract surgery services, the addition of outreach free clinic screening points in the distant districts and addressing of the hidden costs by providing transportation may prove effetive. ${ }^{20}$ Low health awareness in the low-income cohort may be a barrier to seeking healthcare and use of services, including cataract surgery. ${ }^{23}{ }^{27}$ Programme policies should focus on providing intensive counselling and improving the understanding of cataracts through community-based education opportunities to increase the likelihood of seeking surgery. ${ }^{22} 2326$

\section{Strengths and limitations of the study}

This is the first study to summarise the characteristics of the urban poor participating in the free cataract surgery programme, including patient demographics, resources, health conditions, reasons for accepting the free surgery and overall evaluation of the free cataract surgery programme. Several aspects of the telephone survey limit the interpretations of our findings. Firstly, some participants and those who refused to respond may have been reluctant to report their low income and education levels and this may have led to under-reporting even though we had notified all respondents prior to the formal interview that their responses would be protected with strict confidentiality. Another limitation is that this study describes the characteristics of only the low-income cohort that underwent the free cataract surgery in the programme; the characteristics of the low-income patients who did not undergo or have not yet undergone the free cataract surgery represent an area for future research.

\section{Conclusion and implications}

The telephone survey revealed a high level of patient satisfaction regarding the free cataract surgery programme and provided the detailed patient characteristics of this low-income community. Most of the participating patients resided in the major urban districts, and possessed poor health awareness and low education levels. This information is crucial for improving and expanding the management of free cataract surgery programmes in urban China.

Acknowledgements The authors thank Miss Lisha Wang (Shanghai University of Traditional Chinese Medicine, Shanghai, China) for assistance with the figures.

Contributors HL, WC, DL, EL, HJ contributed to the conception or design of the work; HJ, BQ, JT, YL, JC contributed to the acquisition of data; XW, ZL, $X L$, ZL contributed to the analysis; BZ, HC, XT, LL, YL contributed to the interpretation of data for the work; and HL, WC, DL, EL, HJ contributed to drafting of the work and revising it critically for important intellectual content. All authors gave final approval of the version to be published; HL, WC, DL, EL, HJ, YL agree to be accountable for all aspects of the work.

Funding This clinical study was supported by the Pearl River Science and Technology New Star (Grant number 2014J2200060) Project of Guangzhou City, the Guangdong Provincial Natural Science Foundation for Distinguished Young Scholars of China (Grant number 2014A030306030), Youth Science and Technology Innovation Talents Funds in the Special Support Plan for High Level Talents in Guangdong Province (Grant number 2014TQ01R573), the Cultivation Projects (12ykpy61) and the Intensive Cultivation Projects (2015ykzd11) for Young Teaching Staff of Sun Yat-sen University from the Fundamental Research Funds for the Central Universities, the National Natural Science Foundation of China (81300750), the Fundamental Research Funds of State Key Laboratory of Ophthalmology (Grant number 2015QN01) and Key Research Plan for National Natural Science Foundation for Cultivation Project of China (number 91546101). The sponsors of the study played no role in the study protocol design, data collection, data analysis, data interpretation, manuscript preparation, or decision to submit the manuscript for publication.

\section{Competing interests None declared.}

Ethics approval This study was approved by the Human Research Ethics Committee of the ZOC, Sun Yat-sen University. All procedures were consistent with the tenets of the Declaration of Helsinki and were performed after 
obtaining oral informed consent (provision of written consent were exempted by the IRB-ZOC-SYSU) from all respondents on the telephone prior to the formal interview.

Provenance and peer review Not commissioned; externally peer reviewed.

Data sharing statement No additional data are available.

Open Access This is an Open Access article distributed in accordance with the Creative Commons Attribution Non Commercial (CC BY-NC 4.0) license, which permits others to distribute, remix, adapt, build upon this work noncommercially, and license their derivative works on different terms, provided the original work is properly cited and the use is non-commercial. See: http:// creativecommons.org/licenses/by-nc/4.0/

\section{REFERENCES}

1. $\mathrm{Xu} \mathrm{J,} \mathrm{Zhu} \mathrm{S,} \mathrm{Li} \mathrm{S,} \mathrm{et} \mathrm{al.} \mathrm{Models} \mathrm{for} \mathrm{improving} \mathrm{cataract} \mathrm{surgical} \mathrm{rates}$ in southern China. Br J Ophthalmol 2002;86:723-4.

2. Liang YB, Friedman DS, Wong TY, et al. Prevalence and causes of low vision and blindness in a rural Chinese adult population: the Handan Eye Study. Ophthalmology 2008;115:1965-72.

3. Wang $\mathrm{Y}$, Wang J, Maitland E, et al. Growing old before growing rich: inequality in health service utilization among the mid-aged and elderly in Gansu and Zhejiang Provinces, China. BMC Health Serv Res 2012;12:302.

4. Information about national cataract surgery operations in 2014. National health and family planning commission of the people's republic of China. 2015. http://www.moh.gov.cn/yzygj/s7653/201503/ 9aa3cc022c744b28845c121209166491.shtml

5. [No authors listed]. Vision 2020: the cataract challenge. Community Eye Health 2000;13:17-19.

6. VISION 2020 Latin America. Table 1. Cataract surgery rate (per million) and number of ophthalmologists (per million population) for Latin America countries. 2013. http://www.v2020la.org/images/CSR 2012.pdf

7. Zhang XJ, Liang YB, Liu YP, et al. Implementation of a free cataract surgery program in rural China: a community-based randomized interventional study. Ophthalmology 2013;120:260-5

8. Radhakrishnan $M$, Venkatesh $R$, Valaguru $V$, et al. Household preferences for cataract surgery in rural India: a population-based stated preference survey. Ophthalmic Epidemiol 2015;22:34-42.

9. Jiang M, Yang S, Yan K, et al. Measuring access to medicines: a survey of prices, availability and affordability in Shaanxi province of China. PLOS ONE 2013;8:e70836.

10. Yang H, Dib HH, Zhu M, et al. Prices, availability and affordability of essential medicines in rural areas of Hubei Province, China. Health Policy Plan 2010;25:219-29.
11. World Health Organization, Health Action International (2003) Measuring medicine prices, availability, affordability and price components 2nd edition. 2010. http://www.haiweb.org/ medicineprices/manual/documents.html

12. Zhang M, Wu J, Li L, et al. Impact of cataract screening outreach in rural China. Invest Ophthalmol Vis Sci 2010;51:110-14.

13. He M, Chan V, Baruwa E, et al. Willingness to pay for cataract surgery in rural Southern China. Ophthalmology 2007;114:411-16.

14. Baruwa $\mathrm{E}, \mathrm{Tzu} \mathrm{J}$, Congdon $\mathrm{N}$, et al. Reversal in gender valuations of cataract surgery after the implementation of free screening and low-priced high-quality surgery in a rural population of southern China. Ophthalmic Epidemiol 2008;15:99-104.

15. Xi X, Li W, Li J, et al. A survey of the availability, prices and affordability of essential medicines in Jiangsu Province, China. BMC Health Serv Res 2015;15:345.

16. Jiang $\mathrm{H}$, Lin $\mathrm{H}, \mathrm{Qu} \mathrm{B}$, et al. Exploration of management workflow of cataract surgery in an impoverished population in urban China. Eye Sci 2014;29:116-20.

17. World Health Organization. 2014. http://www.who.int/en/

18. Shores KA, West ST. The relationship between built park environments and physical activity in four park locations. J Public Health Manag Pract 2008;14:e9-16.

19. Steven Millward. It's time for messaging apps to quit the bullshit numbers and tell us how many users are active. Techinasia.com. 23 January 2014.

20. Burga HG, Hinds CN, Lansingh VC, et al. Is the cost the primary barrier for cataract surgery in Paraguay? Arq Bras Oftalmol 2014;77:164-7.

21. National health and family planning commission of the people's republic of China. 2015. http://www.nhfpc.gov.cn/zhuzhan/index. shtml

22. Schulze Schwering M, Finger RP, Barrows J, et al. Barriers to uptake of free pediatric cataract surgery in Malawi. Ophthalmic Epidemiol 2014:21:138-43

23. Syed A, Polack S, Eusebio C, et al. Predictors of attendance and barriers to cataract surgery in Kenya, Bangladesh and the Philippines. Disabil Rehabil 2013;35:1660-7.

24. Chen W, Zuo C, Chen C, et al. Prevalence of corneal astigmatism before cataract surgery in Chinese patients. J Cataract Refract Surg 2013;39:188-92.

25. Statistical bulletin about the national economic and social development in Guangzhou. 2013. http://www.gzstats.gov.cn/tjgb/ qstjgb/201304/t20130401_32641.htm

26. Radhakrishnan M, Venkatesh R, Valaguru $\mathrm{V}$, et al. Economic and social factors that influence households not willing to undergo cataract surgery. Indian J Ophthalmol 2015;63:594-9.

27. Mitsuhiro MH, Berezovsky A, Belfort R, et al. Uptake, Barriers and Outcomes in the Follow-up of Patients Referred for Free-of-Cost Cataract Surgery in the Sao Paulo Eye Study. Ophthalmic Epidemio 2015;22:253-9. 